\title{
Expression of eukaryotic translation initiation factor 3 subunit $B$ in liver cancer and its prognostic significance
}

\author{
QING YUE $^{1 *}$, LINGYU MENG $^{2 *}$, BAOXING JIA $^{2}$ and WEI HAN ${ }^{2}$ \\ Departments of ${ }^{1}$ Oncology and ${ }^{2}$ Hepatobiliary and Pancreatic Surgery, \\ The First Hospital of Jilin University, Changchun, Jilin 130021, P.R. China
}

Received July 16, 2019; Accepted December 19, 2019

DOI: $10.3892 /$ etm.2020.8726

\begin{abstract}
Liver cancer is one of the major malignancies with the worst prognosis among all solid tumor types. It is therefore ponderable to explore prognostic biomarkers and therapeutic targets for liver cancer. Eukaryotic translation initiation factor 3 subunit B (EIF3B) is closely linked to the transcription initiation of cancer-associated genes. In the present study, EIF3B was indicated to be a potential prognostic biomarker of liver cancer. The mRNA expression level of EIF3B in liver cancer was assessed by analyzing the Cancer Genome Atlas dataset. $\chi^{2}$ and Fisher's exact tests were used to assess the association of EIF3B expression with clinical parameters. Receiver-operating characteristic curve analysis was used for evaluating the diagnostic value of EIF3B. Overall and relapse-free survival were assessed using Kaplan-Meier curves to determine the association between EIF3B expression and survival. Univariate and multivariate Cox regression analysis were performed to identify the factors affecting overall/relapse-free survival. Gene set enrichment analysis (GSEA) was used to identify signaling pathways associated with EIF3B in liver cancer. It was revealed that EIF3B was highly expressed in liver cancer tissues and it had a promising diagnostic ability. Furthermore, the survival analysis indicated that patients with high EIF3B expression generally had shorter overall as well as relapse-free survival. Univariate and multivariate Cox analysis suggested that high EIF3B mRNA expression may serve as an independent biomarker for the prognostication of patients with liver cancer. GSEA suggested that MYC-V1 (HALLMARK_MYC_TARGETS_V1 geneset; $\mathrm{P}=0.009)$, MYC-V2 (HALLMARK_MYC_TARGETS_V2 geneset; $\mathrm{P}=0.004$ ) and DNA repair pathways (HALLMARK_ DNA_REPAIR geneset; $\mathrm{P}<0.001)$ were differentially enriched
\end{abstract}

Correspondence to: Dr Wei Han, Department of Hepatobiliary and Pancreatic Surgery, The First Hospital of Jilin University, 71 Xinmin Street, Changchun, Jilin 130021, P.R. China

E-mail: weihan_1989@126.com

${ }^{*}$ Contributed equally

Key words: eukaryotic translation initiation factor 3 subunit B, liver cancer, prognosis, The Cancer Genome Atlas, biomarker in high EIF3B expression and low EIF3B expression groups. In conclusion, high EIF3B expression was indicated to be an independent prognostic biomarker for patients with liver cancer.

\section{Introduction}

Liver cancer is a common malignant tumor type with high morbidity and mortality (1). Although various treatments have been improved, the mortality rate of liver cancer is still high and the prognosis remains poor $(2,3)$. Therefore, prognostic biomarkers of liver cancer have become one of the hotspots of current research (4). The discovery of accurate prognostic biomarkers may contribute to clinical guidance in order to improve the evaluation system of liver cancer.

The family of eukaryotic translation initiation factors (EIFs) participates in eukaryotic translation by regulating the interaction between ribosomes and RNA. It is the rate-limiting step of protein synthesis and participates in numerous processes that are deregulated in cancer cells, including DNA repair and proliferation, cell cycle and apoptosis (5). EIF 3 subunit $\mathrm{B}$ (EIF3B) is an important member of the family of EIFs and has been observed to be overexpressed in numerous cancer types, including clear cell renal cell carcinoma (6), esophageal squamous cell carcinoma (7), glioblastoma (8), ovarian cancer (9), osteosarcoma (10) and lung cancer (11), and has an important role in the progression and prognosis of several cancer types (12-14). In addition, Golob-Schwarzl et al (15) reported that EIF3B was upregulated in hepatitis $C$ virus (HCV)-associated hepatocellular carcinoma (HCC). However, the precise role of EIF3B in liver cancer has remained elusive.

To further evaluate the roles of EIF3B in patients with liver cancer, the expression of EIF3B was examined in a dataset from The Cancer Genome Atlas (TCGA) database. The $\chi^{2}$ and Fisher's exact tests were used to assess the association of EIF3B with clinicopathological parameters and demographic features. Receiver operating characteristics (ROC) curve analysis was used for evaluating the diagnostic value of EIF3B. Kaplan-Meier overall survival and relapse-free survival analysis were performed to determine the association between EIF3B expression and survival. Univariate and multivariate Cox regression analysis were performed to identify the factors affecting overall survival and relapse-free survival. Furthermore, gene set enrichment analysis (GSEA) was used to explore EIF3B-associated signaling pathways. 
Table I. Demographic and clinical characteristics of the cohort from The Cancer Genome Atlas-liver hepatocellular carcinoma dataset.

\begin{tabular}{l}
\hline Characteristics \\
\hline Age (years) \\
$<55$ \\
$\geq 55$ \\
NA \\
Sex \\
Female \\
Male \\
Histological type \\
Fibrolamellar carcinoma \\
Hepatocellular carcinoma \\
Hepatocholangiocarcinoma (mixed) \\
Histologic grade \\
NA \\
G1 \\
G2 \\
G3 \\
G4 \\
Stage \\
NA \\
I \\
II \\
III \\
IV
\end{tabular}

T classification

$$
\begin{aligned}
& \text { NA } \\
& \text { T1 } \\
& \text { T2 } \\
& \text { T3 } \\
& \text { T4 } \\
& \text { TX }
\end{aligned}
$$$$
1(0.00)
$$

$121(32.44)$ $252(67.56)$

$$
3(0.80)
$$

7 (1.88)

5 (1.34)

55 (14.75)

178 (47.72)

123 (32.98)

12 (3.22)

24 (6.43)

$172(46.11)$

87 (23.32)

85 (22.79)

5 (1.34)

$2(0.54)$

$182(48.79)$

95 (25.47)

$80(21.45)$

$13(3.49)$

$1(0.27)$

$\mathrm{N}$ classification

$$
\begin{aligned}
& \text { NA } \\
& \text { N0 } \\
& \text { N1 } \\
& \text { NX }
\end{aligned}
$$

M classification

M0

M1

MX

Radiation therapy

$$
\begin{aligned}
& \text { NA } \\
& \text { No } \\
& \text { Yes }
\end{aligned}
$$

253 (67.83)

4 (1.07)

$115(30.83)$

267 (71.58)

4 (1.07)

$102(27.35)$

25 (6.70)

$340(91.15)$

$8(2.14)$

Residual tumor

NA

R0

$\mathrm{R} 1$

$\mathrm{R} 2$

$\mathrm{RX}$
Table I. Continued.

\begin{tabular}{lc}
\hline Characteristics & N (373) \\
\hline Vital status & \\
Deceased & $130(34.85)$ \\
Alive & $243(65.15)$ \\
Sample type & \\
Primary tumor & $371(99.46)$ \\
Recurrent tumor & $2(0.54)$ \\
Overall survival (ten years) & \\
No & $237(64.58)$ \\
Yes & $130(35.42)$ \\
Relapse-free survival (ten years) & $179(55.94)$ \\
No & $141(44.06)$ \\
Yes & \\
EIF3B & $109(29.22)$ \\
High & $264(70.78)$ \\
Low &
\end{tabular}

NA, not available; EIF3B, eukaryotic translation initiation factor 3 subunit B; G1-4, grade relating to degree of differentiation; T1-4, size and or extension of the primary tumor; TX, tumor could not be assessed; N0, no regional lymph node metastasis; N1, regional lymph node metastasis present; NX, lymph nodes could not be assessed; M0, no distant metastasis; M1, metastasis to distant organs; MX, metastasis could not be assessed; R0, no residual tumor visible under the microscope; R1, residual tumor visible under the microscope; R2, residual tumor visible to the naked eye; RX, residual tumor could not be assessed.

\section{Materials and methods}

Data source. The clinical information of patients and their RNAseq data were obtained from TCGA (https://cancergenome.nih.gov/). All patients from the liver hepatocellular carcinoma (LIHC) cohort were screened based on TCGA inclusion and exclusion pre-selection criteria.

Statistical analysis. R (version 3.6.1; The R Foundation) (16) was used for statistical analysis (t-test, Kruskal-Wallis with Dunn's post-hoc test, Wilcoxon sum-rank test) and generation of images. The ggplot2 package (17) was used to draw boxplots of the EIF3B expression in subgroups by clinical characteristics. $\chi^{2}$ and Fisher's exact tests were applied to estimate the significance of the association between EIF3B expression and clinicopathological or demographic characteristics. The pROC package (18) was used to plot the ROC curves and assess the diagnostic ability of EIF3B, and patients were divided into a high expression group and low expression group according to the best operating system cut-off value determined by the Youden index. The survival package (19) was used to draw survival curves. A univariate Cox linear regression model was utilized to select correlative variables affecting survival time. Multivariate Cox regression analysis was employed to evaluate the independent influencing factors of survival time. 
A

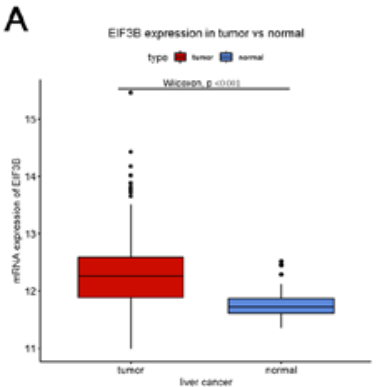

E

E EF3B expersson groupes by histologed ipe

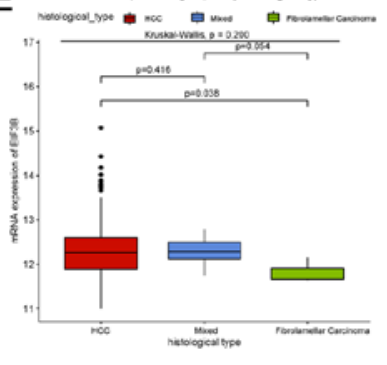

I

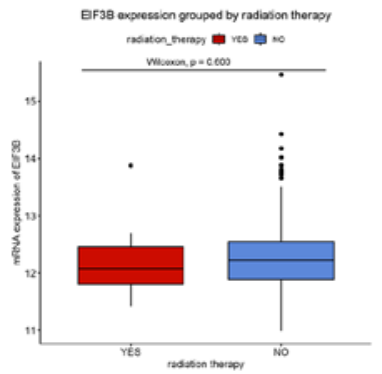

M

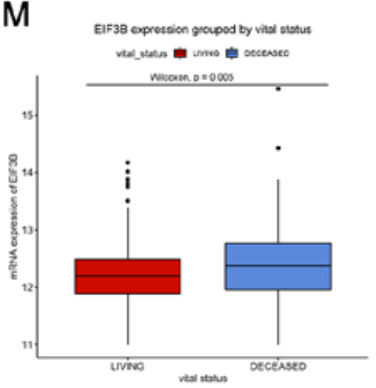

B

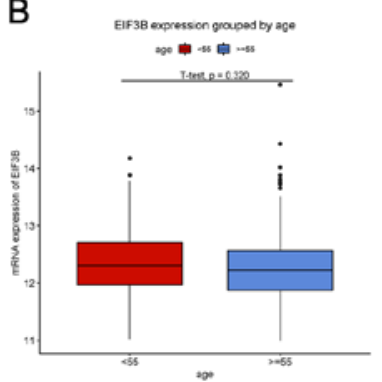

F ElF38 exossion proupod by T classification $\quad$ G

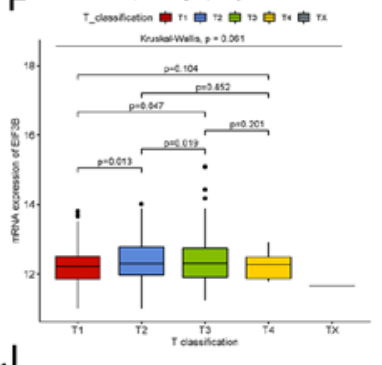

$\mathrm{J}$

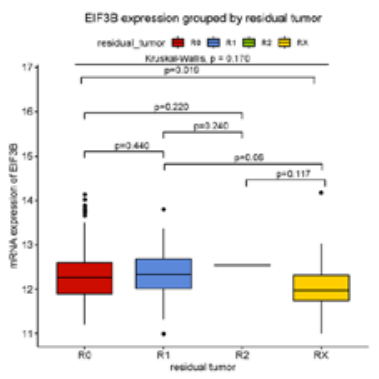

C

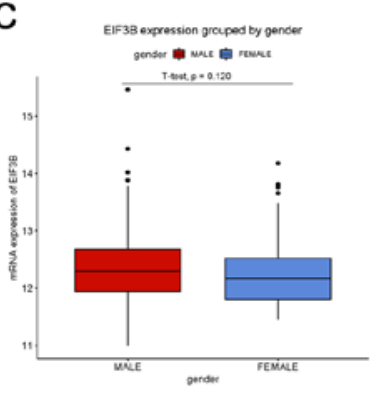

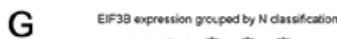

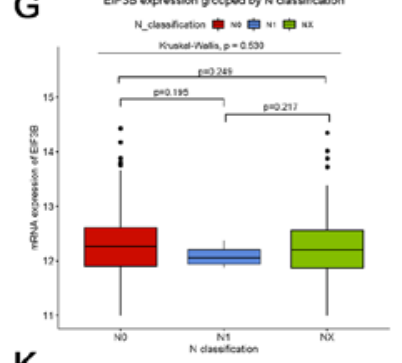

$\mathrm{K}$

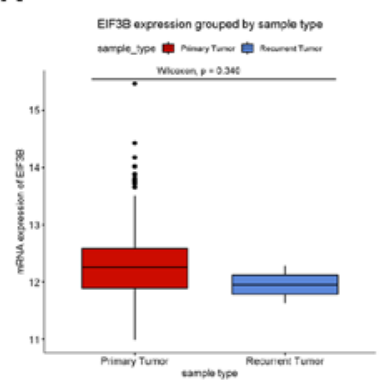

D

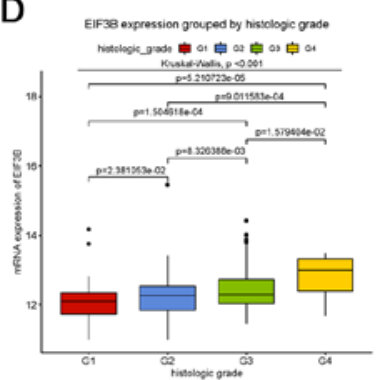

$\mathrm{H}$

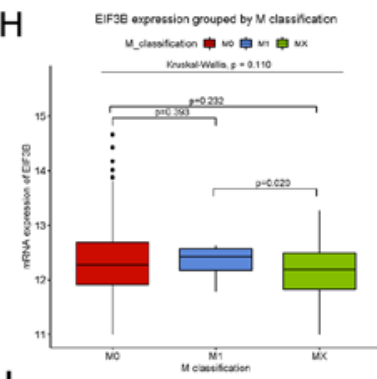

$\mathrm{L}$

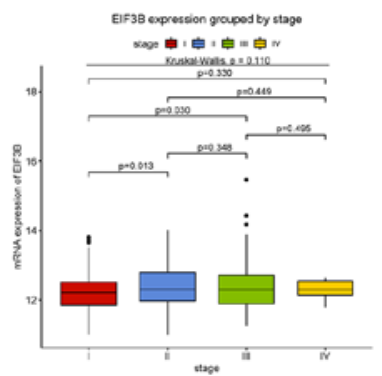

Figure 1. Differences in EIF3B expression among subgroups of patients. Boxplots showing differences in EIF3B expression according to (A) tissue type ( $\mathrm{P}<0.001$ vs. normal tissue controls), (B) age, (C) sex, (D) histologic grade ( $\mathrm{P}<0.001)$, (E) histological type, (F) T classification, (G) N classification, (H) M classification, (I) radiation therapy, (J) residual tumor classification, (K) sample type, (L) clinical stage and (M) vital status ( $\mathrm{P}=0.005)$. EIF3B, eukaryotic translation initiation factor 3 subunit B. G1-4, grade relating to degree of differentiation; T1-4, size and or extension of the primary tumor; TX, tumor could not be assessed; N0, no regional lymph node metastasis; N1, regional lymph node metastasis present; NX, lymph nodes could not be assessed; M0, no distant metastasis; M1, metastasis to distant organs; MX, metastasis could not be assessed; R0, no residual tumor visible under the microscope; R1, residual tumor visible under the microscope; R2, residual tumor visible to the naked eye; RX, residual tumor could not be assessed.

GSEA. GSEA may be used to determine whether a predefined set of genes is able to indicate significant, consistent differences between two biological states (20). In the present study, GSEA was performed in the 'h.all.v6.2.symbols.gmt' and 'c2. cp.biocarta.v6.2.symbols.gmt' gene sets using GSEA3.0 software. The standardized enrichment fraction was obtained by 1,000 permutation analyses.

\section{Results}

Patient characteristics. In Table I, the clinical features of the 373 patient cohort, including sex (female 121, male 252), age (16-90 years old, median 61, mean 59.47), use of radiation therapy, residual tumor, relapse-free survival, histological type, stage, vital status, survival data, T/N/M classification and EIF3B expression are provided.

Expression of EIF3B in liver tissues. Boxplots revealed that EIF3B was significantly upregulated in liver cancer compared with that in normal liver tissues (Fig. 1A; $<<0.001$ ). Furthermore, EIF3B was also differentially expressed between subgroups by vital status $(\mathrm{P}=0.005)$ and histologic grade $(\mathrm{P}<0.001 ;$ Fig. 1$)$.

Diagnostic capability of EIF3B. To assess the diagnostic performance of EIF3B in liver cancer, ROC curve analysis was used. The area under the curve (AUC) was 0.821, indicating 
Table II. Association between the expression of EIF3B and the clinicopathological characteristics of patients with liver cancer.

\begin{tabular}{|c|c|c|c|c|c|}
\hline \multirow[b]{2}{*}{ Clinical characteristics } & \multirow[b]{2}{*}{ No. of patients } & \multicolumn{2}{|c|}{ EIF3B expression } & \multirow[b]{2}{*}{$\chi^{2}$} & \multirow[b]{2}{*}{ P-value } \\
\hline & & High & Low & & \\
\hline Age (years) & & & & 0.089 & 0.765 \\
\hline$<55$ & 117 & $36(33.03)$ & $81(30.80)$ & & \\
\hline$\geq 55$ & 255 & $73(66.97)$ & $182(69.20)$ & & \\
\hline Sex & & & & 0.484 & 0.486 \\
\hline Female & 121 & $32(29.36)$ & $89(33.71)$ & & \\
\hline Male & 252 & $77(70.64)$ & $175(66.29)$ & & \\
\hline Histological type & & & & 1.251 & 0.534 \\
\hline Fibrolamellar carcinoma & 3 & $0(0.00)$ & $3(1.14)$ & & \\
\hline Hepatocellular carcinoma & 363 & $107(98.17)$ & $256(96.97)$ & & \\
\hline Hepatocholangiocarcinoma (mixed) & 7 & $2(1.83)$ & $5(1.89)$ & & \\
\hline Histologic grade & & & & 17.796 & $<0.001$ \\
\hline G1 & 55 & $9(8.33)$ & $46(17.69)$ & & \\
\hline $\mathrm{G} 2$ & 178 & 45 (41.67) & $133(51.15)$ & & \\
\hline G3 & 123 & $46(42.59)$ & 77 (29.62) & & \\
\hline G4 & 12 & $8(7.41)$ & $4(1.54)$ & & \\
\hline Stage & & & & 4.532 & 0.209 \\
\hline I & 172 & $43(40.95)$ & $129(52.87)$ & & \\
\hline II & 87 & $32(30.48)$ & $55(22.54)$ & & \\
\hline III & 85 & $28(26.67)$ & $57(23.36)$ & & \\
\hline IV & 5 & $2(1.9)$ & $3(1.23)$ & & \\
\hline $\mathrm{T}$ classification & & & & 7.720 & 0.102 \\
\hline $\mathrm{T} 1$ & 182 & $44(40.37)$ & $138(52.67)$ & & \\
\hline $\mathrm{T} 2$ & 95 & 34 (31.19) & $61(23.28)$ & & \\
\hline T3 & 80 & $29(26.61)$ & $51(19.47)$ & & \\
\hline $\mathrm{T} 4$ & 13 & $2(1.83)$ & $11(4.2)$ & & \\
\hline TX & 1 & $0(0.00)$ & $1(0.38)$ & & \\
\hline $\mathrm{N}$ classification & & & & 1.936 & 0.379 \\
\hline No & 253 & 77 (70.64) & $176(66.92)$ & & \\
\hline N1 & 4 & $0(0.00)$ & $4(1.52)$ & & \\
\hline NX & 115 & $32(29.36)$ & $83(31.56)$ & & \\
\hline M classification & & & & 2.882 & 0.236 \\
\hline M0 & 267 & $83(76.15)$ & $184(69.70)$ & & \\
\hline M1 & 4 & $2(1.83)$ & $2(0.76)$ & & \\
\hline MX & 102 & $24(22.02)$ & $78(29.55)$ & & \\
\hline Radiation therapy & & & & $<0.001$ & $>0.999$ \\
\hline No & 340 & $92(97.87)$ & 248 (97.64) & & \\
\hline Yes & 8 & $2(2.13)$ & $6(2.36)$ & & \\
\hline Residual tumor & & & & 5.116 & 0.163 \\
\hline R0 & 326 & 98 (91.59) & $228(88.03)$ & & \\
\hline $\mathrm{R} 1$ & 17 & $5(4.67)$ & $12(4.63)$ & & \\
\hline $\mathrm{R} 2$ & 1 & $1(0.93)$ & $0(0.00)$ & & \\
\hline $\mathrm{RX}$ & 22 & $3(2.80)$ & $19(7.34)$ & & \\
\hline Vital status & & & & 17.505 & $<0.001$ \\
\hline Deceased & 130 & $56(51.38)$ & $74(28.03)$ & & \\
\hline Alive & 243 & $53(48.62)$ & 190 (71.97) & & \\
\hline Sample type & & & & 0.017 & 0.895 \\
\hline Primary tumor & 371 & $109(100)$ & $262(99.24)$ & & \\
\hline Recurrent tumor & 2 & $0(0.00)$ & $2(0.76)$ & & \\
\hline
\end{tabular}


Table II. Continued.

\begin{tabular}{lcccrr}
\hline & & \multicolumn{2}{c}{ EIF3B expression } & & \\
Clinical characteristics & No. of patients & High & Low & $\chi^{2}$ & P-value \\
\hline Overall survival (ten years) & & & & 18.690 & $<0.001$ \\
$\quad$ No & 237 & $50(47.17)$ & $187(71.65)$ & & \\
$\quad$ Yes & 130 & $56(52.83)$ & $74(28.35)$ & & 0.312 \\
Relapse-free survival (ten years) & 179 & $42(50.6)$ & $137(57.81)$ & & \\
$\quad$ No & 141 & $41(49.4)$ & $100(42.19)$ & & \\
Yes & & & & \\
\hline
\end{tabular}

EIF3B, eukaryotic translation initiation factor 3 subunit B; G1-4, grade relating to degree of differentiation; T1-4, size and or extension of the primary tumor; TX, tumor could not be assessed; N0, no regional lymph node metastasis; N1, regional lymph node metastasis present; NX, lymph nodes could not be assessed; M0, no distant metastasis; M1, metastasis to distant organs; MX, metastasis could not be assessed; R0, no residual tumor visible under the microscope; R1, residual tumor visible under the microscope; R2, residual tumor visible to the naked eye; $\mathrm{RX}$, residual tumor could not be assessed.
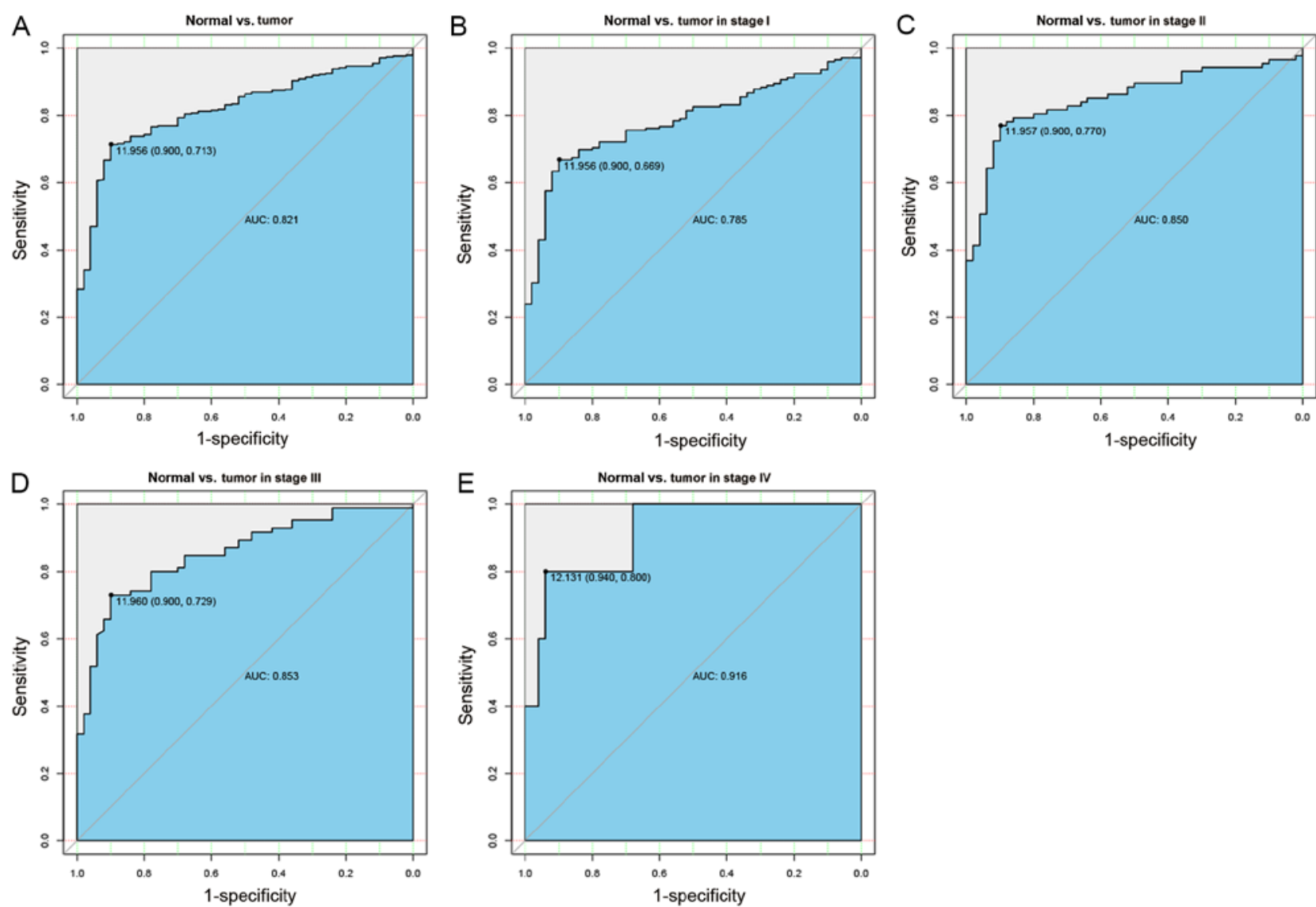

Figure 2. Receiver-operating characteristic curves for EIF3B in The Cancer Genome Atlas-liver hepatocellular carcinoma dataset. The ability of EIF3B to distinguish between the following was assessed: (A) Non-tumor vs. tumor sample; non-tumor sample vs. tumor sample of (B) stage I, (C) stage II, (D) stage III and (E) stage IV. AUC, area under the curve; EIF3B, eukaryotic translation initiation factor 3 subunit B.

that EIF3B has moderate diagnostic ability. In addition, similar AUCs were obtained for distinguishing normal liver tissues from liver cancer at specific stages (stage I, 0.785; stage II, 0.850; stage III, 0.853; stage IV, 0.916; Fig. 2).

Association between EIF3B expression and clinical features of patients with liver cancer. As indicated in Table II, the vital status of the patients with liver cancer $(\mathrm{P}<0.001)$, overall survival ( $\mathrm{P}<0.001$; duration ten years) and the histologic grade $(\mathrm{P}<0.001)$ were associated with the expression of EIF3B.

High expression of EIF3B is associated with poor overall survival of patients with liver cancer. Kaplan-Meier analysis indicated that high expression of EIF3B was significantly 

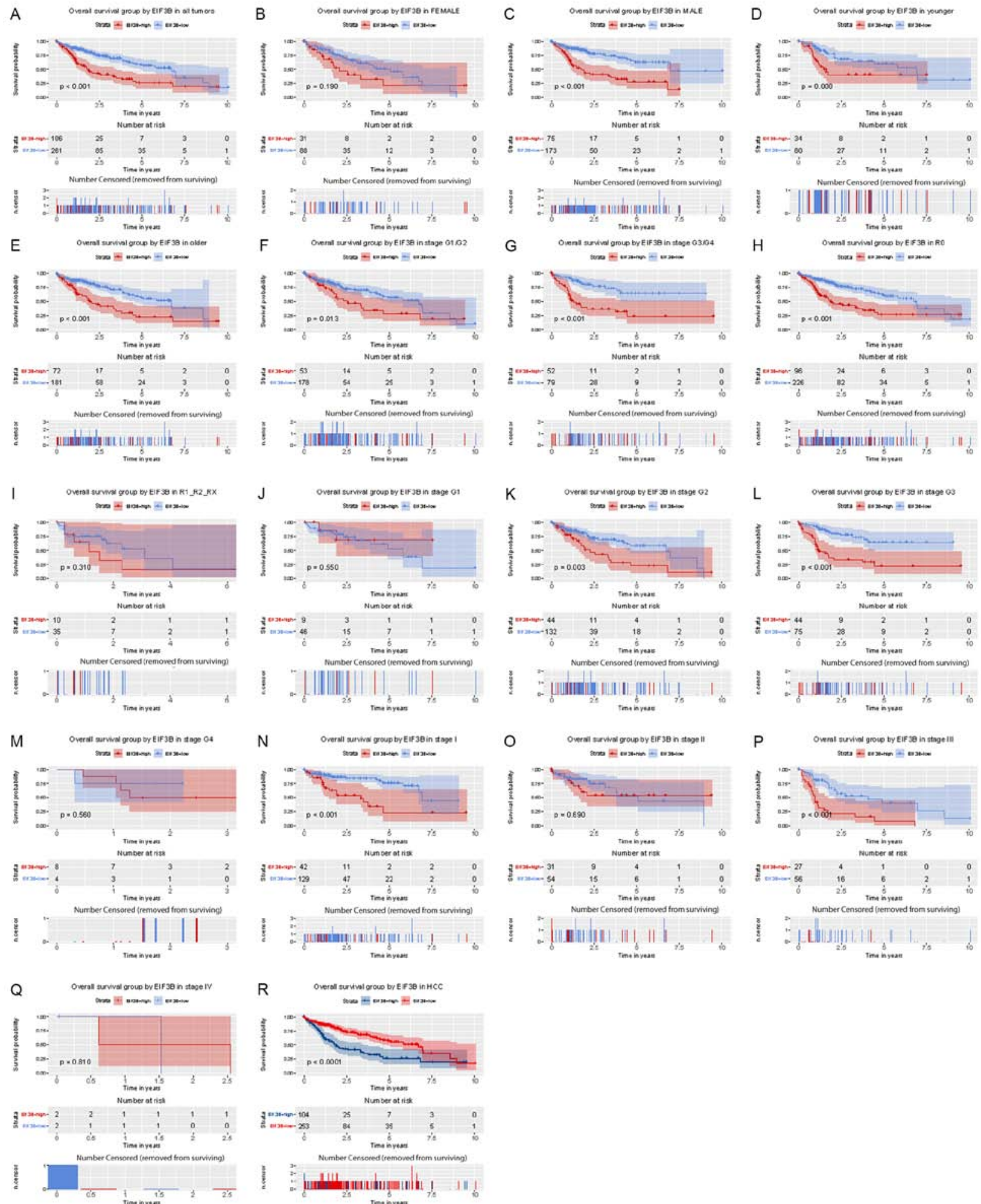

Figure 3. Kaplan-Meier analysis of the influence of EIF3B expression on overall survival. (A) All patients. Subgroup analysis for (B) females, (C) males, (D) younger patients (<55), (E) older patients ( $\geq 55)$, (F) no lymph node dissection (R0), (G) lymph node dissection (R1/R2/RX), (H-M) histological grade, (H) G1/G2, (I) G3/G4, (J) G1, (K) G2, (L) G3, (M) G4, (N-Q) clinical stage (N) I, (O) II, (P) III, (Q) IV and (R) HCC. EIF3B, eukaryotic translation initiation factor 3 subunit B; HCC, hepatocellular carcinoma; G1-4, grade relating to degree of differentiation; T1-4, size and or extension of the primary tumor; TX, tumor could not be assessed; N0, no regional lymph node metastasis; N1, regional lymph node metastasis present; NX, lymph nodes could not be assessed; M0, no distant metastasis; M1, metastasis to distant organs; MX, metastasis could not be assessed; R0, no residual tumor visible under the microscope; R1, residual tumor visible under the microscope; R2, residual tumor visible to the naked eye; RX, residual tumor could not be assessed. The number of results censored (removed from surviving) is indicated below the survival curve.

associated with poor overall survival $(\mathrm{P}<0.001$; Fig. 3$)$. Subgroup analysis provided similar results, particularly in female $(\mathrm{P}<0.001)$, younger $(<55 ; \mathrm{P}=0.008)$ and older subjects $(\geq 55 ; \mathrm{P}<0.001)$, and in patients with $\mathrm{R} 0(\mathrm{P}<0.001)$, $\mathrm{G} 2(\mathrm{P}=0.003), \mathrm{G} 3(\mathrm{P}<0.0001)$, stage I $(\mathrm{P}<0.001)$ and stage III $(\mathrm{P}<0.001)$. 

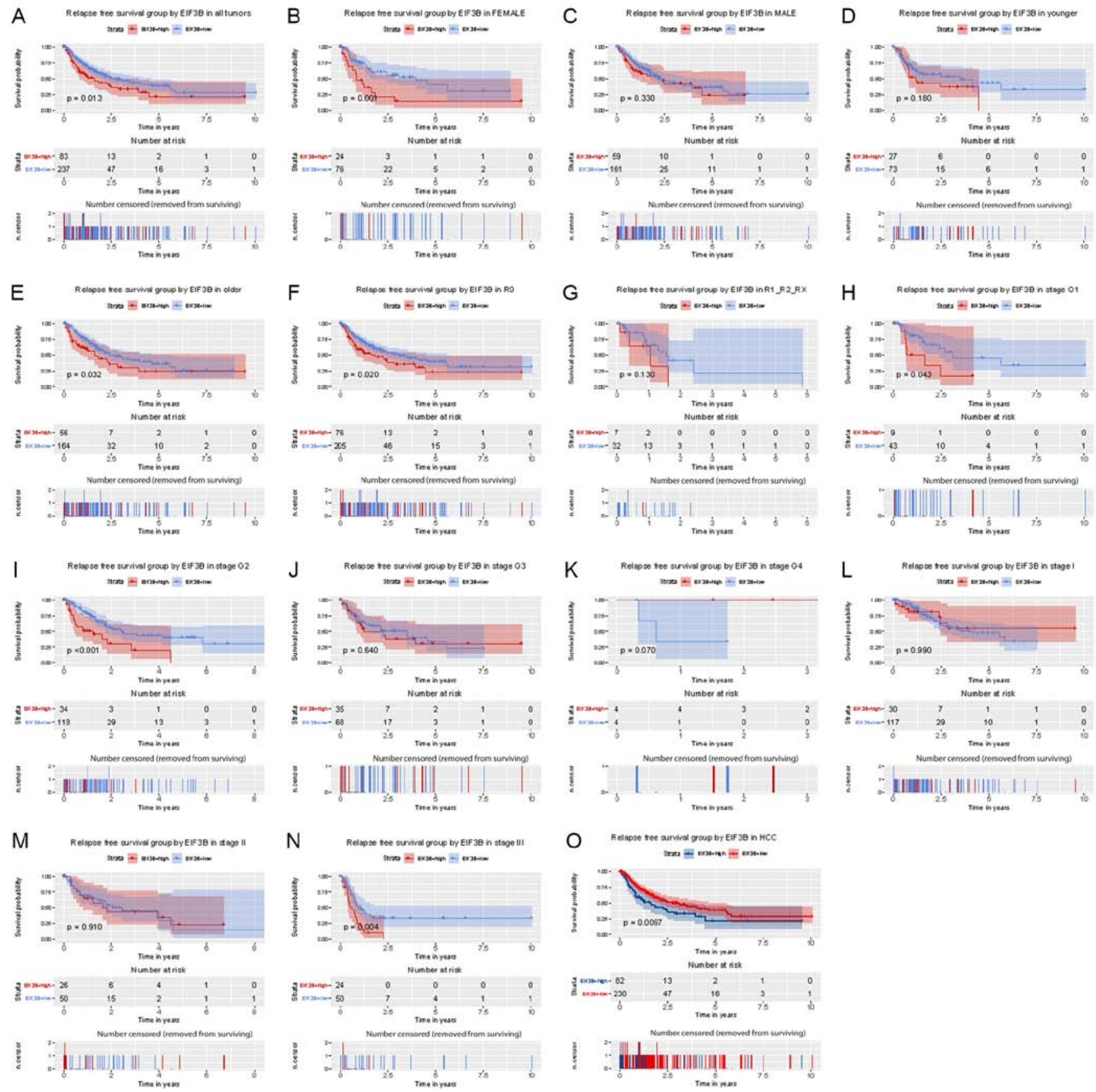

Figure 4. Kaplan-Meier analysis of the influence of EIF3B expression on relapse-free survival. (A) All patients. (B-R) Subgroup analysis for (B) females, (C) males, (D) younger patients (<55), (E) older patients ( $\geq 55),(\mathrm{F})$ no lymph node dissection (R0), (G) lymph node dissection (R1/R2/RX), (H-K) histological grade (H) G1, (I) G2, (J) G3, (K) G4, (L-N) clinical stage (L) I, (M) II, (N) III and (O) HCC. EIF3B, eukaryotic translation initiation factor 3 subunit B; HCC, hepatocellular carcinoma; G1-4, grade relating to degree of differentiation; T1-4, size and or extension of the primary tumor; TX, tumor could not be assessed; N0, no regional lymph node metastasis; N1, regional lymph node metastasis present; NX, lymph nodes could not be assessed; M0, no distant metastasis; M1, metastasis to distant organs; MX, metastasis could not be assessed; R0, no residual tumor visible under the microscope; R1, residual tumor visible under the microscope; R2, residual tumor visible to the naked eye; RX, residual tumor could not be assessed. The number of results censored (removed from surviving) is indicated below the survival curve.

As presented in Table III, T classification, stage, residual tumor and EIF3B expression were variables associated with overall survival according to univariate Cox regression analysis. In addition, multivariate Cox regression indicated that high EIF3B expression, $\mathrm{T}$ classification and residual tumor were independent risk factors for overall survival of patients with liver cancer [hazard ratio $(\mathrm{HR})=2.44,95 \% \mathrm{CI}=1.71-3.47$, $\mathrm{P}<0.001]$.
High expression of EIF3B is associated with poor relapse-free survival of patients with liver cancer. Kaplan-Meier analysis indicated that patients with high expression of EIF3B had significantly poorer relapse-free survival ( $\mathrm{P}=0.013$; Fig. 4). Subgroup analysis provided similar results, particularly in female $(\mathrm{P}=0.001)$ and older $(\geq 55 ; \mathrm{P}=0.032)$ subjects and in patients with $\mathrm{R} 0(\mathrm{P}=0.020), \mathrm{G} 2(\mathrm{P}<0.001)$ and stage III $(\mathrm{P}=0.004)$. 
Table III. Summary of univariate and multivariate Cox regression analyses for overall survival duration (ten years).

\begin{tabular}{|c|c|c|c|c|c|c|}
\hline \multirow[b]{2}{*}{ Parameters } & \multicolumn{3}{|c|}{ Univariate analysis } & \multicolumn{3}{|c|}{ Multivariate analysis } \\
\hline & Hazard ratio & $95 \% \mathrm{CI}$ & P-value & Hazard ratio & $95 \% \mathrm{CI}$ & P-value \\
\hline Age $(\geq 55 /<55$ years $)$ & 1.00 & $0.69-1.45$ & 0.997 & & & \\
\hline Sex (male/female) & 0.80 & $0.56-1.14$ & 0.220 & & & \\
\hline \multicolumn{7}{|l|}{ Histological type (hepatocholangiocarcinoma/ } \\
\hline Hepatocellular, Hepatocellular /fibrolamellar) & 0.99 & $0.27-3.66$ & 0.986 & & & \\
\hline Histologic grade (G4/G3/G2/G1) & 1.04 & $0.84-1.30$ & 0.698 & & & \\
\hline Stage (IV/III/II/I) & 1.38 & $1.15-1.66$ & 0.001 & 0.81 & $0.65-1.01$ & 0.060 \\
\hline T classification (T4/T3/T2/T1/NX) & 1.66 & $1.39-1.99$ & $<0.001$ & 1.91 & $1.51-2.42$ & $<0.001$ \\
\hline $\mathrm{N}$ classification $(\mathrm{N} 1 / \mathrm{N} 0 / \mathrm{NX})$ & 0.73 & $0.51-1.05$ & 0.086 & & & \\
\hline M classification (M1/M0/MX) & 0.72 & $0.49-1.04$ & 0.077 & & & \\
\hline Radiation therapy (yes/no) & 0.51 & $0.26-1.03$ & 0.060 & & & \\
\hline Residual tumor classification (RX/R2/R1/R0) & 1.42 & $1.13-1.80$ & 0.003 & 1.45 & $1.13-1.87$ & 0.004 \\
\hline EIF3B (high/low) & 2.41 & $1.70-3.42$ & $<0.001$ & 2.44 & $1.71-3.47$ & $<0.001$ \\
\hline
\end{tabular}

EIF3B, eukaryotic translation initiation factor 3 subunit B; G1-4, grade relating to degree of differentiation; T1-4, size and or extension of the primary tumor; TX, tumor could not be assessed; N0, no regional lymph node metastasis; N1, regional lymph node metastasis present; NX, lymph nodes could not be assessed; M0, no distant metastasis; M1, metastasis to distant organs; MX, metastasis could not be assessed; R0, no residual tumor visible under the microscope; R1, residual tumor visible under the microscope; R2, residual tumor visible to the naked eye; $\mathrm{RX}$, residual tumor could not be assessed.

Table IV. Summary of univariate and multivariate Cox regression analyses or relapse-free survival duration.

\begin{tabular}{|c|c|c|c|c|c|c|}
\hline \multirow[b]{2}{*}{ Parameters } & \multicolumn{3}{|c|}{ Univariate analysis } & \multicolumn{3}{|c|}{ Multivariate analysis } \\
\hline & Hazard ratio & $95 \% \mathrm{CI}$ & P-value & Hazard ratio & $95 \% \mathrm{CI}$ & P-value \\
\hline Age $(\geq 55 /<55$ years $)$ & 0.90 & $0.63-1.28$ & 0.550 & & & \\
\hline Sex (male/female) & 0.99 & $0.70-1.41$ & 0.966 & & & \\
\hline Histological type (hepatocholangiocarcinoma/ & & & & & & \\
\hline hepatocellular, hepatocellular /fibrolamellar) & 2.02 & $0.66-6.24$ & 0.220 & & & \\
\hline Histologic grade (G4/G3/G2/G1) & 0.98 & $0.80-1.21$ & 0.883 & & & \\
\hline Stage (IV/III/II/I) & 1.66 & $1.38-1.99$ & $<0.001$ & 1.10 & $0.85-1.42$ & 0.473 \\
\hline $\mathrm{T}$ classification $(\mathrm{T} 4 / \mathrm{T} 3 / \mathrm{T} 2 / \mathrm{T} 1 / \mathrm{TX})$ & 1.78 & $1.49-2.12$ & $<0.001$ & 1.67 & $1.28-2.18$ & $<0.001$ \\
\hline $\mathrm{N}$ classification $(\mathrm{N} 1 / \mathrm{N} 0 / \mathrm{NX})$ & 0.97 & $0.67-1.40$ & 0.874 & & & \\
\hline M classification (M1/M0/MX) & 1.17 & $0.79-1.74$ & 0.432 & & & \\
\hline Radiation therapy (yes/no) & 0.74 & $0.26-2.16$ & 0.584 & & & \\
\hline Residual tumor classification (RX/R2/R1/R0) & 1.28 & $1.01-1.61$ & 0.042 & 1.36 & $1.07-1.73$ & 0.012 \\
\hline EIF3B (high/low) & 1.58 & $1.10-2.28$ & 0.014 & 1.54 & $1.06-2.23$ & 0.022 \\
\hline
\end{tabular}

EIF3B, eukaryotic translation initiation factor 3 subunit B; G1-4, grade relating to degree of differentiation; T1-4, size and/or extension of the primary tumor; TX, tumor could not be assessed; N0, no regional lymph node metastasis; N1, regional lymph node metastasis present; NX, lymph nodes could not be assessed; M0, no distant metastasis; M1, metastasis to distant organs; MX, metastasis could not be assessed; R0, no residual tumor visible under the microscope; R1, residual tumor visible under the microscope; R2, residual tumor visible to the naked eye; $\mathrm{RX}$, residual tumor could not be assessed.

As presented in Table IV, T classification, stage, residual tumor and EIF3B expression were variables associated with relapse-free survival according to the univariate Cox regression analysis. In addition, high EIF3B expression, $\mathrm{T}$ classification and residual tumor were independent risk factors for relapse-free survival of patients with liver cancer in the multivariate Cox regression analysis $(\mathrm{HR}=1.54,95 \%$ $\mathrm{CI}=1.06-2.23, \mathrm{P}=0.022$ ).

Signaling pathways associated with EIF3B. To identify the signaling pathways associated with EIF3B in liver cancer, GSEA was performed between the low EIF3B expression 
Table V. Gene sets enriched in phenotype high.

Molecular signatures

database collection

Gene set name

NES

NOM P-value

FDR q-value

h.all.v6.2.symbols.gmt

h.all.v6.2.symbols.gmt

HALLMARK_MYC_TARGETS_V2

2.154

0.004

0.006

h.all.v6.2.symbols.gmt

HALLMARK_MYC_TARGETS_V1

2.028

0.009

0.010

HALLMARK_DNA_REPAIR

2.006

$<0.001$

0.009

Gene sets with NOM P-value $<0.050$ and FDR q-value $<0.250$ were considered as significant. FDR, false discovery rate; NES, normalized enrichment score; NOM, nominal.
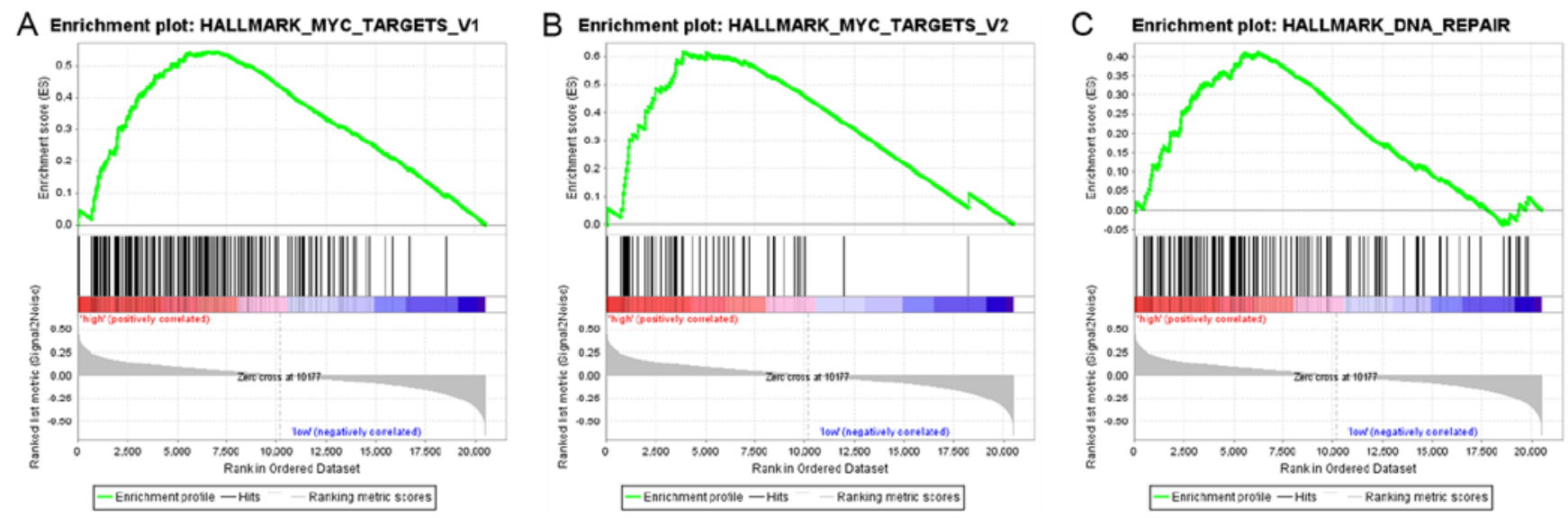

Figure 5. Enrichment plots from GSEA. The GSEA results indicated that (A) the MYC-V1, (B) the MYC-V2 and (C) the DNA repair pathway are differentially enriched in high EIF3B expression and low EIF3B expression groups. GSEA, gene set enrichment analysis.

dataset and the high EIF3B expression dataset. The enrichment of the molecular signatures database (MSigDB) determined by GSEA was significantly different (nominal P-value $<0.050$, false discovery rate $<0.250$; Table V). As presented in Fig. 5 and Table V, GSEA indicated that MYC-V1 (HALLMARK MYC_TARGETS_V1 geneset; $\mathrm{P}=0.009)$, MYC-V2 (HALLMARK_MYC_TARGETS_V2 geneset; $\mathrm{P}=0.004$ ) and DNA repair pathways (HALLMARK_DNA_REPAIR geneset; $\mathrm{P}<0.001$ ) were differentially enriched in high EIF3B expression and low EIF3B expression groups.

\section{Discussion}

Liver cancer is associated with a high mortality rate worldwide; the development of this cancer type may be influenced by viral infection, diet and environmental factors (21-24). In recent years, with the continuous progression of molecular biology and treatments, including chemotherapeutic drugs and surgical technology, the understanding of cancer biology and the treatment of liver cancer have made great progress. However, the prognosis of liver cancer remains poor. The World Health Organization/International Classification of Diseases-10 classifies diseases according to their etiology, pathology, clinical manifestations and anatomical location. Cancer is a gene-associated disease and molecular typing is required to deepen our understanding of the underlying mechanisms of disease development (25). Therefore, novel biomarkers are urgently required. Our research group has been exploring novel biomarkers for a number of years (26-38). The present study focused on EIF3B and indicated that EIF3B is a potential and independent prognostic biomarker for liver cancer.

EIF3B is closely linked to cancer progression. Consistent with previous studies, it was indicated that EIF3B was highly expressed in patients with liver cancer. Although Golob-Schwarzl et al (15) reported that EIF3B was upregulated in $\mathrm{HCV}$-associated $\mathrm{HCC}$, all of their patients were Asians. The patients assessed in the present study were from all over the world and covered other types of liver cancer that may be related to $\mathrm{HCV}$. All of these results indicate that EIF3B has an important role in cancer-associated processes. A previous study suggested that EIF3B is involved in the proliferation and metastasis of gastric cancer (39). In addition, the present study further determined that EIF3B is associated with the histologic grade and survival status of patients with liver cancer. Therefore, in-depth studies using experimental and bioinformatics methods are required.

EIF3B has been indicated to have a marked influence on the prognosis of patients with cancer. In the present study, it was observed that upregulation of EIF3B was associated with poor overall/relapse-free survival of patients with liver cancer. In addition, patients with high EIF3B expression in clear cell renal cell carcinoma, esophageal squamous cell carcinoma and non-small cell lung cancer had a shorter survival time $(6,7,11)$. In order to further explore the association between EIF3B and clinical characteristics of patients with liver cancer, a subgroup analysis was performed. As the TCGA-LIHC dataset does not have a Barcelona Clinic Liver Cancer staging system, TNM 
staging was used. Kaplan-Meier subgroup analysis indicated that high expression of EIF3B was associated with poor overall survival in the subgroups of females, younger $(<55)$ or older ( $\geq 55$ ) patients, R0, G2, G3, stage I and stage III. Furthermore, high expression of EIF3B was associated with poor relapse-free survival in the subgroups of females, older patients, R0, G2 and stage III. However, Tian et al (11) reported that upregulation of EIF3B was associated with tumor depth, TNM stage and lymph node metastasis in patients with esophageal squamous cell carcinoma. However, the results of the present study indicated that EIF3B was related to histological grade and survival status. This may be linked to the heterogeneity of tumor types and individual differences, which may help to select personalized treatments. Owing to the TCGA-LIHC data not including the body mass index and the presence of diabetes mellitus as variables, it is not possible to calculate their association with the prognosis of patients.

In the GSEA analysis, high EIF3B expression was indicated to be associated with MYC-V1, MYC-V2 and DNA repair in liver cancer. The MYC oncogene is an important regulator of liver cancer progression. Previous studies have indicated that MYC is able to promote the proliferation, metastasis and metabolism of liver cancer by regulating signaling pathways including AKT/mTOR and RAS/mitogen-activated protein kinase (40-42). In addition, each replication of DNA in cancer cells may cause a large amount of damage, including DNA substitutions or deletions (43). Therefore, DNA repair mechanisms (damage induction, signal transduction, signal response) are particularly important. This may explain why EIF3B may promote the progression of liver cancer through MYC-V1/V2 and DNA repair pathways.

The present study mainly uncovered the prognostic value of the EIF3B mRNA expression in liver cancer. Along with other studies on EIF3B, the present study contributed to a better understanding of the role of EIF3B, as well as the great possibility for precise prognostication. However, the underlying mechanisms remain to be fully elucidated and require further exploration by scientific research. In the future, the mechanisms of EIF3B will be studied at a deeper level.

In conclusion, the present study investigated the prognostic value of EIF3B in patients with liver cancer. High EIF3B expression was proved to be a potential and independent prognostic biomarker for liver cancer. Future work will include in vivo and in vitro experiments to explore the biological functions of EIF3B and the underlying mechanisms.

\section{Acknowledgements}

Not applicable.

\section{Funding}

No funding was received.

\section{Availability of data and materials}

Patient information was obtained from the open TCGA database (https://portal.gdc.cancer.gov/). The datasets used and/or analyzed during the current study are available from the corresponding author on reasonable request.

\section{Authors' contributions}

WH designed the study. QY and LM were responsible for extracting data, conducted data analysis and wrote the first draft of the manuscript. BJ participated in the analysis of data and critical modification of important knowledge content. WH and BJ critically revised the manuscript and gave final approval for submission. All authors read and approved the final manuscript.

\section{Ethics approval and consent to participate}

Not applicable.

\section{Patient consent for publication}

Not applicable.

\section{Competing interests}

The authors declare that they have no competing interests.

\section{References}

1. Siegel RL, Miller KD and Jemal A: Cancer statistics, 2019. CA Cancer J Clin 69: 7-34, 2019.

2. Ryerson AB, Eheman CR, Altekruse SF, Ward JW, Jemal A, Sherman RL,Henley SJ,Holtzman D,Lake A, Noone AM, et al: Annual Report to the Nation on the Status of Cancer, 1975-2012, Featuring the Increasing Incidence of Liver Cancer. Cancer 122: 1312-1337, 2016.

3. Li L and Wang H: Heterogeneity of liver cancer and personalized therapy. Cancer Lett 379: 191-197, 2016.

4. Dawkins J and Webster RM: The hepatocellular carcinoma market. Nat Rev Drug Discov 18: 13-14, 2019.

5. Smith MD, Arake-Tacca L, Nitido A, Montabana E, Park A and Cate JH: Assembly of eIF3 mediated by mutually dependent subunit insertion. Structure 24: 886-896, 2016.

6. Zang Y,Zhang X, Yan L, Gu G, LiD,Zhang Y, Fang L, Fu S, Ren J and $\mathrm{Xu} \mathrm{Z}$ : Eukaryotic translation initiation factor $3 \mathrm{~b}$ is both a promising prognostic biomarker and a potential therapeutic target for patients with clear cell renal cell carcinoma. J Cancer 8: 3049-3061, 2017.

7. Xu F, Xu CZ, Gu J, Liu X, Liu R, Huang E, Yuan Y, Zhao G, Jiang $\mathrm{J}, \mathrm{Xu} \mathrm{C}$, et al: Eukaryotic translation initiation factor 3B accelerates the progression of esophageal squamous cell carcinoma by activating $\beta$-catenin signaling pathway. Oncotarget 7 : 43401-43411, 2016

8. Liang H, Ding X, Zhou C, Zhang Y, Xu M, Zhang C and Xu L: Knockdown of eukaryotic translation initiation factors $3 \mathrm{~B}$ (EIF3B) inhibits proliferation and promotes apoptosis in glioblastoma cells. Neurol Sci 33: 1057-1062, 2012.

9. Wang L and Ouyang L: Effects of EIF3B gene downregulation on apoptosis and proliferation of human ovarian cancer SKOV3 and HO-8910 cells. Biomed Pharmacother 109: 831-837, 2019.

10. Choi YJ, Lee YS, Lee HW, Shim DM and Seo SW: Silencing of translation initiation factor eIF3b promotes apoptosis in osteosarcoma cells. Bone Joint Res 6: 186-193, 2017.

11. Tian Y, Zhao K, Yuan L, Li J, Feng S, Feng Y, Fang Z, Li H and Deng R: EIF3B correlates with advanced disease stages and poor prognosis, and it promotes proliferation and inhibits apoptosis in non-small cell lung cancer. Cancer Biomark 23: 291-300, 2018.

12. Spilka R, Ernst C, Mehta AK and Haybaeck J: Eukaryotic translation initiation factors in cancer development and progression. Cancer Lett 340: 9-21, 2013.

13. Wang H, Ru Y, Sanchez-Carbayo M, Wang X, Kieft JS and Theodorescu D: Translation initiation factor eIF3b expression in human cancer and its role in tumor growth and lung colonization. Clin Cancer Res 19: 2850-2860, 2013.

14. Lin L, Holbro T, Alonso G, Gerosa D and Burger MM: Molecular interaction between human tumor marker protein p150, the largest subunit of eIF3, and intermediate filament protein K7. J Cell Biochem 80: 483-490, 2001. 
15. Golob-Schwarzl N, Krassnig S, Toeglhofer AM, Park YN, Gogg-Kamerer M, Vierlinger K, Schröder F, Rhee H, Schicho R, Fickert $\mathrm{P}$ and Haybaeck J: New liver cancer biomarkers: $\mathrm{PI} 3 \mathrm{~K} / \mathrm{AKT} / \mathrm{mTOR}$ pathway members and eukaryotic translation initiation factors. Eur J Cancer 83: 56-70, 2017.

16. Team RDCJC: R: A language and environment for statistical computing. R Foundation for Statistical Computing, Vienna, Austria. 14: 12-21, 2009.

17. Wickham H: Ggplot2: Elegant graphics for data analysis. J R Stat Soc 174: 245-246, 2011

18. Robin X, Turck N, Hainard A, Tiberti N, Lisacek F, Sanchez JC and Müller M: pROC: An open-source package for R and S+ to analyze and compare ROC curves. BMC Bioinformatics 12: 77, 2011.

19. Therneau TM and Grambsch PM: Modeling survival data: Extending the Cox model. Springer, New York, 2000.

20. Subramanian A, Tamayo P, Mootha VK, Mukherjee S, Ebert BL, Gillette MA, Paulovich A, Pomeroy SL, Golub TR, Lander ES and Mesirov JP: Gene set enrichment analysis: A knowledge-based approach for interpreting genome-wide expression profiles. Proc Natl Acad Sci USA 102: 15545-15550, 2005.

21. Cronin KA, Lake AJ, Scott S, Sherman RL, Noone AM Howlader N, Henley SJ, Anderson RN, Firth AU, Ma J, et al: Annual report to the nation on the status of cancer, part I: National cancer statistics. Cancer 124: 2785-2800, 2018.

22. Xu N, Liu YN, Yin P, Wang LJ, Dou YS, Yang WJ and Zhou MG: Impact of liver cancer deaths on life expectancy in 14 counties (districts) from the Huai River Basin, 2013: Relationship between the water environment and liver cancer. Zhonghua Yu Fang Yi Xue Za Zhi 50: 629-633, 2016 (In Chinese).

23. Tu T, Bühler S and Bartenschlager R: Chronic viral hepatitis and its association with liver cancer. Biol Chem 398: 817-837, 2017.

24. Chen YJ, Wallig MA and Jeffery EH: Dietary broccoli lessens development of fatty liver and liver cancer in mice given diethylnitrosamine and fed a western or control diet. J Nutr 146: 542-550, 2016.

25. Global Burden of Disease Cancer Collaboration, Fitzmaurice C, Akinyemiju TF, Al Lami FH, Alam T, Alizadeh-Navaei R, Allen C, Alsharif U, Alvis-Guzman N, Amini E, et al: Global, regional, and national cancer incidence, mortality, years of life lost, years lived with disability, and disability-adjusted life-years for 29 cancer groups, 1990 to 2016: A systematic analysis for the global burden of disease study. JAMA Oncol 4: 1553-1568, 2018.

26. Jiao Y, Fu Z, Li Y, Meng L and Liu Y: High EIF2B5 mRNA expression and its prognostic significance in liver cancer: A study based on the TCGA and GEO database. Cancer Manag Res 10: 6003-6014, 2018.

27. Jiao Y, Fu Z, Li Y, Zhang W and Liu Y: Aberrant FAM64A mRNA expression is an independent predictor of poor survival in pancreatic cancer. PLoS One 14: e0211291, 2019.

28. Jiao Y, Li Y, Lu Z and Liu Y: High trophinin-associated protein expression is an independent predictor of poor survival in liver cancer. Dig Dis Sci 64: 137-143, 2019.

29. Jiao Y, Li Y, Fu Z, Hou L, Chen Q, Cai Y, Jiang P, He M and Yang Z: OGDHL expression as a prognostic biomarker for liver cancer patients. Dis Markers 2019: 9037131, 2019.
30. Jiao Y, Li Y, Jiang P, Han W and Liu Y: PGM5: A novel diagnostic and prognostic biomarker for liver cancer. PeerJ 7: e7070, 2019.

31. Jiao Y, Li Y, Liu S, Chen Q and Liu Y: ITGA3 serves as a diagnostic and prognostic biomarker for pancreatic cancer. Onco Targets Ther 12: 4141-4152, 2019.

32. Li Y, Jiao Y, Fu Z, Luo Z, Su J and Li Y: High miR-454-3p expression predicts poor prognosis in hepatocellular carcinoma. Cancer Manag Res 11: 2795-2802, 2019.

33. Li Y, Jiao Y, Li Y and Liu Y: Expression of La ribonucleoprotein domain family member 4B (LARP4B) in liver cancer and their clinical and prognostic significance. Dis Markers 2019: 1569049, 2019.

34. Li Y, Jiao Y, Luo Z, Li Y and Liu Y: High peroxidasin-like expression is a potential and independent prognostic biomarker in breast cancer. Medicine (Baltimore) 98: e17703, 2019.

35. Zhang X, Cui Y, He M, Jiao Y and Yang Z: Lipocalin-1 expression as a prognosticator marker of survival in breast cancer patients. Breast Care, 2019.

36. Cui Y, Jiao Y, Wang K, He M and Yang Z: A new prognostic factor of breast cancer: High carboxyl ester lipase expression related to poor survival. Cancer Genet 239: 54-61, 2019.

37. Hou L, Zhang X, Jiao Y, Li Y, Zhao Y, Guan Y and Liu Z: ATP binding cassette subfamily B member 9 (ABCB9) is a prognostic indicator of overall survival in ovarian cancer. Medicine (Baltimore) 98: e15698, 2019.

38. Cai H, Jiao Y, Li Y, Yang Z, He M and Liu Y: Low CYp24A1 mRNA expression and its role in prognosis of breast cancer. Sci Rep 9: 13714, 2019.

39. Ma F, Li X, Ren J, Guo R, Li Y, Liu J, Sun Y, Liu Z, Jia J and $\mathrm{Li}$ W: Downregulation of eukaryotic translation initiation factor $3 \mathrm{~b}$ inhibited proliferation and metastasis of gastric cancer. Cell Death Dis 10: 623, 2019

40. Ladu S, Calvisi DF, Conner EA, Farina M, Factor VM and Thorgeirsson SS: E2F1 inhibits c-Myc-driven apoptosis via pIK3CA/Akt/mTOR and COX-2 in a mouse model of human liver cancer. Gastroenterology 135: 1322-1332, 2008.

41. Xin B, Yamamoto M, Fujii K, Ooshio T, Chen X, Okada Y, Watanabe K, Miyokawa N, Furukawa H and Nishikawa Y: Critical role of Myc activation in mouse hepatocarcinogenesis induced by the activation of AKT and RAS pathways. Oncogene 36: 5087-5097, 2017.

42. Li X, Wu Q, Bu M, Hu L, Du WW, Jiao C, Pan H, Sdiri M, Wu N, Xie Y and Yang BB: Ergosterol peroxide activates Foxo3-mediated cell death signaling by inhibiting AKT and c-Myc in human hepatocellular carcinoma cells. Oncotarget 7: 33948-33959, 2016.

43. Barnes JL, Zubair M, John K, Poirier MC and Martin FL: Carcinogens and DNA damage. Biochem Soc Trans 46: 1213-1224, 2018

(i) $($ This work is licensed under a Creative Commons Attribution-NonCommercial-NoDerivatives 4.0 International (CC BY-NC-ND 4.0) License. 